\title{
Quality of treatment and surgical approach for rectal gastrointestinal stromal tumour (GIST) in a large European cohort
}

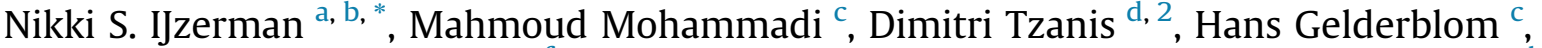 \\ Marco Fiore ${ }^{\mathrm{e}}$, Elena Fumagalli ${ }^{\mathrm{f}}$, Piotr Rutkowski ${ }^{\mathrm{g}}$, Elzbieta Bylina ${ }^{\mathrm{g}}$, Ioannis Zavrakidis ${ }^{\mathrm{h}}$, \\ Neeltje Steeghs ${ }^{a}$, Han J. Bonenkamp ${ }^{\mathrm{i}}$, Boudewijn van Etten ${ }^{\mathrm{j}}$, Dirk J. Grünhagen ${ }^{\mathrm{k}}$, \\ Shahnawaz Rasheed ${ }^{1}$, Paris Tekkis ${ }^{l}$, Charles Honoré ${ }^{\mathrm{m}, 2}{ }^{2}$, Winan van Houdt ${ }^{\mathrm{n}}$, \\ Jos van der Hage $^{\circ}$, Sylvie Bonvalot ${ }^{\mathrm{d}, 2}$, Yvonne Schrage ${ }^{\mathrm{p}, 1}$, Myles Smith ${ }^{\mathrm{n}, 1}$ \\ a Sarcoma Unit, Department of Medical Oncology, Netherlands Cancer Institute, Amsterdam, the Netherlands \\ ${ }^{\mathrm{b}}$ Department of Medical Oncology, Erasmus MC Cancer Institute, Erasmus University Medical Center, Rotterdam, the Netherlands \\ ${ }^{c}$ Leiden University Medical Center, Department of Medical Oncology, Leiden, the Netherlands \\ d Department of Surgery, Institut Curie, PSL University, Paris, France ${ }^{2}$ \\ e Fondazione IRCCS Istituto Nazionale dei Tumori, Department of Surgery, Milano, Italy \\ ${ }^{\mathrm{f}}$ Fondazione IRCCS Istituto Nazionale dei Tumori, Department of Medical Oncology, Milano, Italy \\ g Maria Sklodowska-Curie National Research Institute of Oncology, Department of Soft Tissue/Bone Sarcoma and Melanoma, Warsaw, Poland \\ ${ }^{\mathrm{h}}$ Netherlands Cancer Institute - Antoni van Leeuwenhoek, Department of Epidemiology and Biostatistics, Amsterdam, the Netherlands \\ ${ }^{\mathrm{i}}$ Radboud University Medical Centre, Department of Surgical Oncology, Nijmegen, the Netherlands \\ ${ }^{\mathrm{j}}$ University of Groningen, University Medical Centre Groningen, Department of Surgery, Groningen, the Netherlands \\ ${ }^{\mathrm{k}}$ Erasmus MC - Cancer Institute, Department of Surgical Oncology, Rotterdam, the Netherlands \\ ${ }^{1}$ Sarcoma Unit, Department of Surgical Oncology, Royal Marsden Hospital, London, UK \\ ${ }^{\mathrm{m}}$ Gustave Roussy, Surgical Oncology department, France ${ }^{2}$ \\ ${ }^{\mathrm{n}}$ Netherlands Cancer Institute - Antoni van Leeuwenhoek, Department of Surgical Oncology, Amsterdam, the Netherlands \\ o Leiden University Medical Center, Department of Surgery, Leiden, the Netherlands \\ p Netherlands Cancer Institute - Antoni van Leeuwenhoek, Department of Surgical Oncology, the Netherlands and European School of Soft Tissue Sarcoma \\ Surgery, Amsterdam, the Netherlands
}

\section{A R T I C L E I N F O}

Article history:

Received 14 January 2020

Accepted 20 February 2020

Available online $\mathrm{xxx}$

\section{Keywords:}

Gastrointestinal stromal tumours

Rectum

Surgery

Treatment outcome

Survival

\begin{abstract}
A B S T R A C T
Background: Rectal gastrointestinal stromal tumours (GISTs) are rare tumours. Variability in the management may influence outcome, but there is a lack of understanding regarding contemporary variance in care. A multicenter, international, retrospective cohort study was performed to elucidate characteristics and outcomes of rectal GIST in European practice, with particular reference to surgical approach. Methods: All rectal GIST patients diagnosed between 2009 and 2018 were identified from five European databases. Recurrence free survival (RFS) and overall survival (OS) were estimated using Kaplan-Meier method. Possible confounders were identified using Cox regression analyses.

Results: From 210 patients, 155 patients had surgery. The three main types of surgery were local tumour resection (LTR, $\mathrm{n}=46$ ), low anterior resection (LAR, $\mathrm{n}=31$ ) and abdomino-perineal resection (APR, $\mathrm{n}=32$ ). Most patients received neoadjuvant $(65 \%)$ and/or adjuvant imatinib therapy (66\%). Local recurrence rate after surgery was $15 \%$ and overall recurrence rate $28 \%$. No significant differences were found in terms of RFS nor OS between LTR, LAR and APR. However, locally resected tumours were smaller, while LAR and APR patients more often received perioperative imatinib. General hospitals treated smaller GISTs, offered imatinib less frequently, and had a higher tumour rupture rate. In the
\end{abstract}

\footnotetext{
* Corresponding author. Netherlands Cancer Institute, Department of Medical Oncology, Plesmanlaan 121, 1066, CX, Amsterdam, Netherlands.

E-mail addresses: n.ijzerman@nki.nl (N.S. IJzerman), m.mohammadi@lumc.nl (M. Mohammadi), dimitri.tzanis@curie.fr (D. Tzanis), a.j.gelderblom@lumc.nl

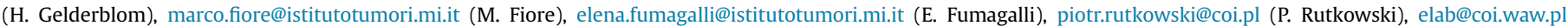

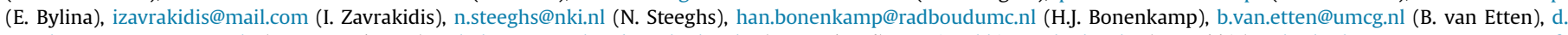

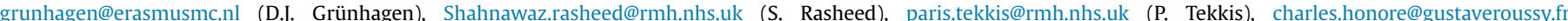

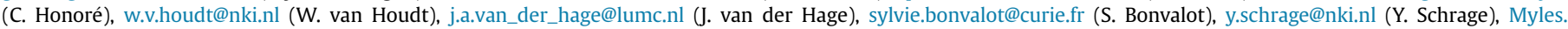
Smith@rmh.nhs.uk (M. Smith).

1 these authors share senior authorship.

2 on behalf of the French Sarcoma group.
} 
multivariate analysis in the group having LTR, APR or LAR, the only significant prognostic factor for local recurrence was higher age (HR 1.06, CI 1.00-1.12, $\mathrm{p}=0.048$ ).

Conclusions: In European clinical practice for rectal GIST, LTR, LAR and APR have comparable local control. Multimodal approach is higher and tumour rupture less frequent in specialist centres compared to general hospitals.

๑ 2020 Elsevier Ltd, BASO The Association for Cancer Surgery, and the European Society of Surgical Oncology. All rights reserved.

\section{Introduction}

Gastrointestinal stromal tumours (GISTs) are rare tumours arising from the mesenchymal tissue in the gastrointestinal tract with an estimated incidence of 10-15 per million per year[1]. Approximately $5 \%$ of the GISTs occur in the rectum[2-5]. Variability in the management of this rare tumour may influence outcome, but there is a lack of understanding regarding contemporary variance in care. Due to the rarity of GIST, a multidisciplinary approach with the involvement of centres with relevant expertise has been recognized as an important factor[6]. There is however limited evidence-based data regarding the treatment of rectal GIST. Most published studies are either single centre experiences with limited number of patients[7-12] or series lacking detailed data about important prognostic factors[13,14], which makes it difficult to interpret the data and limits the clinical application.

Furthermore, since GISTs located in the rectum has been described as an adverse prognostic factor[15], the European Society of Medical Oncology (ESMO) guidelines state that surgical resection should be considered in all rectal GIST patients, irrespectively of tumour size[6]. Furthermore, MRI is advised as primary imaging modality. However, specific surgical strategies are not discussed within ESMO guidelines. Consideration of the surgical approach, is important due to the technically challenging nature of rectal GIST, relating to the precise site of origin in the rectum, its relation to the sphincter complex, the risk of tumour rupture and positive margins, and the relationship to vital structures in the pelvic cavity. The decision about the approach and extent of surgery is therefore crucial for achieving histologically negative margins and at the same time it should be balanced according both to oncological risk and functional morbidity. Other considerations in this group of patients are related to the mode of biopsy, the influence and timing of neoadjuvant treatment on mode of surgery and oncological outcomes, and whether there is benefit in being managed in a highvolume specialist centre.

To address some of these questions, we present a multicentre, international retrospective study including patients with rectal GIST from five European countries, which represents one of the largest series in rectal GIST. The primary aim of this study is to elucidate the characteristics of rectal GIST and outcome of different treatment modalities in contemporary European practice.

\section{Methods}

\subsection{Study design and patient selection}

This is a retrospective, multicenter, international cohort study. We adhered to the STROBE guidelines for cohort studies. Data from five different GIST databases were combined to achieve an adequate sample size. All patients diagnosed with rectal GIST between January 2009 and January 2018 were selected to prevent any type of selection bias. Participating countries were the Netherlands (Dutch GIST consortium, sites: UMC Groningen, Netherlands Cancer Institute, Leiden UMC, Erasmus MC, Radboud MC), Italy (site:
Fondazione IRCSS Istituto Nazionale dei Tumori Milano), France (French Sarcoma Group, sites: NetSarc centres), UK (site: the Royal Marsden Hospital) and Poland (site: Maria Sklodowska-Curie National Research Institute of Oncology). Patients were excluded from the analysis when essential information was missing, being gender and whether patients underwent surgery.

\subsection{Main outcome measures}

The primary endpoint was to describe the outcomes of rectal GIST in European practice, in terms of local recurrence rate, recurrence free survival (RFS) and disease specific survival (DSS). Secondary descriptive endpoints were tumour characteristics (i.e. size, mitotic count, mutational status, baseline distance to anal verge), the impact of neoadjuvant and adjuvant imatinib therapy (i.e. time to maximal tumour reduction, change in size and mitotic count, percentage of post-treatment viable cells), number of patients with radiotherapy and surgery characteristics (i.e. type of surgery, severe complications classified by at least grade $3 \mathrm{~b}$ according to ClavienDindo classification, margins, peroperative tumour rupture).

\subsection{Surgical procedures}

Local tumour resection (LTR) includes transanal excision, transanal endoscopic microsurgery and transperineal approach for resection of the anal canal. Two other common surgical procedures were low anterior resection (LAR) and abdomino-perineal resection (APR).

\subsection{Statistical analyses}

Statistical analyses were performed using IBM SPSS Statistics 25. Time to maximum reduction of tumour size on neoadjuvant imatinib treatment was calculated from start date until the date of the maximum achieved radiological reduction. Survival estimates were obtained using the Kaplan-Meier method and compared by logrank test. RFS was calculated from date of surgery to date of recurrence or date of last follow-up. DSS was calculated from the date of diagnosis to date of death or date of last follow-up. Potential confounders were identified by using univariate Cox regression analysis. Only confounders with a p-value below 0.3 were subsequently included in the multivariate analysis. All tests were twosided and a p-value of $<0.05$ was considered significant. In the group with the three main types of surgery, multiple imputation (Predictive Mean Matching, 20 times, 50 iterations) was performed in SPSS for missing values of likely confounders.

\section{Results}

\subsection{Patient characteristics}

In total, 231 patients with rectal GIST were identified from the databases. After exclusion of 21 patients due missing essential information, 210 patients were ultimately included: 48 patients from 
the Netherlands, 35 from Italy, 22 from the UK, 36 from Poland and 69 from France.

Median age in these 210 patients was 61 years with $63 \%$ being male (Table A1). Median tumour size at diagnosis was $65 \mathrm{~mm}$ and median baseline distance to anal verge $34 \mathrm{~mm}$. Three most common reported symptoms at diagnosis were rectal mass, rectal bleeding and change in bowel habit. Mutation status was known for 156 patients (74\%). Most of the GISTs were KIT exon 11 mutated (71\%), but also mutations in KIT exon 9 (14\%), KIT exon 13 (3\%), PDGFR $(\mathrm{n}=1)$ and KIT/PDGFR wildtype (12\%) were reported. Mitotic rate at baseline was low ( $\leq 5 / 50 \mathrm{HPF})$ in $59 \%$ and high $(>5 / 50 \mathrm{HPF})$ in $41 \%$ of the patients.

In this cohort, 55 patients did not undergo surgery (Fig. A1). The main reason for this was metastatic disease, other reasons were unfitness for surgery, patients declining surgery and ongoing response on systemic treatment. From the surgeries, LTR was most often performed, followed by APR and LAR.

\subsection{Main types of surgery}

The group with the three main types of surgeries (LTR, LAR and APR) consists of 109 patients (Table A2). None of these patients had metastatic disease at diagnosis. Median age in this group was 61 years and 70\% were male. Median baseline tumour size was $61 \mathrm{~mm}$ and median distance to the anal verge $35 \mathrm{~mm}$. In one patient, the tumour extended into the sphincter and in 4 patients it was $\leq 1 \mathrm{~cm}$ from the sphincter. Most GISTs were KIT exon 11 mutated (74\%). Comparing the three groups of surgery, smaller tumours more often had LTR. No significant differences were found in baseline mitotic rate, peroperative tumour rupture and resection margins. The severe complication rate was low (4.4\%). Patients with a LAR or APR received neoadjuvant imatinib therapy more frequently compared to the LTR group ( $77 \%$ or $91 \%$ vs. $54 \%$ resp.), but this difference was not noticed in the adjuvant treated group ( $86 \%$ or $61 \%$ vs. $61 \%$ resp., $\mathrm{p}=0.062$ ). When comparing LAR with APR separately, patients with a LAR received adjuvant imatinib more often than patients having an APR (86\% vs. 61\%). No other differences were found in characteristics between patients with a LAR and APR.

\subsection{Location of surgery: general hospital versus specialist centre}

For 140 patients that had surgery (90\%) the type of hospital was specified. Fifty-one (36\%) operations were performed in a general hospital not specialized in GIST surgery. These operations were mainly LTRs and baseline tumour size was smaller (median $44 \mathrm{~mm}$ vs. median $67 \mathrm{~mm}, \mathrm{p}=0.002$ ). Patients that had surgery in general hospitals were less often treated with neoadjuvant and adjuvant imatinib ( $13 \%$ vs. $96 \%$ ( $\mathrm{p}<0.001)$ and $51 \%$ vs. $72 \%$ ( $\mathrm{p}=0.026)$ resp.). No difference was found in resection margins $(p=0.131)$, but peroperative tumour rupture was more often reported in general hospitals compared to specialized centres ( $37 \%$ vs. $5 \%$, p < 0.001 ). Local RFS was significantly shorter for all patients with peroperative tumour rupture $(\mathrm{n}=17)$ in univariate $K M$ analysis $(\mathrm{p}<0.001$, median not reached), but this effect was lost in multivariate Cox regression analysis. Furthermore, no statistically significant difference was detected for local RFS between patients that underwent surgery in a general hospital or specialist centre $(p=0.240$, median not reached).

\section{4. (Neo)adjuvant treatment}

In the surgery cohort, most of the patients received neoadjuvant (65\%) and/or adjuvant therapy (66\%). Neoadjuvant therapy was imatinib $400 \mathrm{mg}$ QD in 91\% of the cases, and few received imatinib $800 \mathrm{mg}$ QD ( $\mathrm{n}=7$, mostly KIT exon 9 mutated $)$ or masitinib or sunitinib if there was severe imatinib toxicity $(n=2$ and $n=1$ resp.). Median time on neoadjuvant treatment was 10 months (range 1-102), whereas the time to maximum tumour reduction, reviewed retrospectively and available for only $45 \%$ of patients, was 6 months (median, range 2-38). Patients treated with neoadjuvant imatinib had a median size reduction of 33\% (range -100 to $20 \%$, available for $86 \%$ of the patients) and a decrease in mitotic count of 2.5 (median, range -39 to 11 , only available for $36 \%$ of the patients). The median percentage of viable cells after neoadjuvant therapy was $30 \%(0-100$, available for $51 \%$ of the patients). Median time on adjuvant imatinib treatment was 25 months (range 0-112). In the whole cohort at least 12 patients received radiotherapy. Four patients were treated with radiotherapy adjuvant because of high risk features and, remarkably, only one of them had a recurrence.

\subsection{Outcome in general}

Median follow-up time after surgery was 28 months (range $0-115)$. During this follow-up time 43 patients had a recurrence (recurrence rate 28\%): 17 patients a local recurrence (recurrence rate $11 \%), 19$ patients had distant recurrent disease only (recurrence rate $12 \%$ ) and 7 patients had simultaneously local and distant recurrent disease (recurrence rate 5\%). Median all RFS was 75 months (95\% confidence interval (CI) of 64-85 months). Median local RFS was not reached. Overall 12 patients died from which 10 died of disease (5\%), but most of them did not have surgery: only 3 patients that underwent surgery died of disease (2\%). There was no difference in DSS $(\mathrm{p}=0.644)$ comparing the three main types of surgery.

\subsection{Prognostic factors for a local recurrence within the group with three main surgeries}

Using the Kaplan Meier method, local RFS did not differ between the three main surgeries (Fig. A2). No difference in local RFS was found comparing the country of surgery $(\mathrm{p}=0.348)$. Furthermore, local RFS was not significantly longer after adjuvant imatinib $(\mathrm{p}=0.848)$ nor neoadjuvant imatinib $(\mathrm{p}=0.186)$. No difference in local RFS was found comparing high risk rectal GIST (size $>5 \mathrm{~cm}$, mitotic count $>5$ and/or peroperative tumour rupture) with low risk rectal GIST $(\mathrm{p}=0.283)$.

Cases with missing data were automatically excluded from the Cox regression analysis. To make the results more reliable, multiple imputation of the missing data was done for the expected possible confounders within the group of three main surgeries (percentage missing data: baseline mitotic rate $42 \%$, distance to anal verge $25 \%$, peroperative tumour rupture $21 \%$, severe complications $17 \%$ and mutation status 16\%). After multiple imputation, Cox regression analysis was performed (Table A3). Using the p-value threshold of 0.3 , older age, larger baseline tumour size, closer to anal verge, positive resection margin, peroperative tumour rupture and no neoadjuvant imatinib were associated with worse local RFS on univariate Cox regression analysis. In multivariate analysis, using a p-value threshold of 0.05 , the only significant prognostic factor for local recurrence was older age (HR 1.057, CI 1.000-1.116, $\mathrm{p}=0.048$ ).

\section{Discussion}

In this retrospective cohort study, the characteristics of rectal GIST in European practice were investigated. The average rectal GIST patient in this series is 61 years old and male (63\%), presenting most commonly with a rectal mass, rectal bleeding or a change in bowel habits. The vast majority has a resection of the primary tumour, being most frequently a LTR, LAR or APR. The recurrence rate after surgery was high, despite the majority of patients having 
perioperative treatment with imatinib. Smaller tumours were most likely treated by LTR, while LAR and APR patients received more often perioperative imatinib therapy. LTR, LAR and APR appear to have comparable oncological outcome.

Several European studies relating to rectal GIST have been published [7-10], all focusing on differences in outcome after surgery with and without neoadjuvant imatinib, but these studies suffer from small sample sizes and may not reflect contemporary practice. Two recent studies were performed with a larger sample size, but are limited by lack of detailed data about important prognostic factors, tumour size prior to pre-operative therapy and recurrence rate $[14,16]$. Our cohort has the benefit of having a larger sample size, representing multiple institutions, with a wide range of variables that may help inform practice.

Contrary to expectations, in our cohort, tumour rupture and negative resection margins did not appear to influence the risk for recurrence in multivariate analysis. A possible explanation could be a protective effect of perioperative imatinib, but this did not appear to reduce the risk for local recurrence in multivariate analyses either. That may be related both to selection bias in treating patients at higher risk with imatinib and to the relatively low number of events.

The median time of maximum tumour reduction on neoadjuvant imatinib in our series was 6 months. This duration is in line with the time to maximum tumour reduction of 6.9 months found by Wang et al. in 17 rectal GIST patients[17]. Despite the dogma that optimal neoadjuvant imatinib therapy takes longer in rectal GIST patients, the duration of treatment to maximal tumour regression seems comparable to other GIST locations[18,19]. Furthermore, the range of optimal response time is wide and frequent scans to evaluate response should be scheduled.

In this cohort, twelve patients had radiotherapy. Due to the overall good response rates to imatinib, reliable data about the influence of radiotherapy is scarce[6]. In recent years however, several studies were performed regarding radiotherapy in GIST. Joensuu et al. investigated radiotherapy in 25 metastatic GIST patients, whereof 19 patients were concomitantly treated with tyrosine kinase inhibitors. Responses to radiotherapy were infrequent (8\%), but most patients had durable stabilization (80\%) despite confounding from TKI treatment is likely[20]. Some other series show promising results in specific scenarios, but more studies are needed to determine the specific influence of radiotherapy on oncological outcome[21-23]. However, radiotherapy can be considered among possible therapeutic options in patients who do not want surgery and wish to stop or are progressive on TKIs.

Consideration should be given to the type of institution initial surgery was performed, and if there is any potential influence on outcomes. We observed that despite the smaller tumour size in general hospitals, the frequency of tumour rupture was higher in general hospitals compared to specialist centres. This suggests that the quality of surgery was superior in specialist centres: despite smaller cancers and less extensive surgery, there was a higher rate of tumour rupture in general hospitals. Moreover, patients had lower chance to be offered multimodal treatment in general hospitals. Nevertheless, the oncological outcome was not different in general hospitals, which might be explained by selection bias where the high risk tumours are referred to specialized centres. These findings highlight the importance of management of rectal GISTs in specialist centres. The overall rarity of rectal GIST determines that they are very unfamiliar in routine clinical care. Management is frequently complex and pathways of care are complicated, which can be overcome by specialized multidisciplinary GIST care. Moreover, another advantage of centralized care is that more patients can be included in studies which will ultimately ensure improved care.
Of interest was that a substantial proportion of patients declined surgery when indicated. Declining end stomas and concerns regarding and the impact of surgery on quality of life were identified as the most common underlying motivations. This demonstrates the importance of shared decision making with patients and personalized surgery for patients with rectal GIST, in particular with the availability of alternative treatments to surgical resection.

The ESMO guideline recommends MRI as pre-operative imaging in rectal GIST. However, when collecting the data, we noticed that in the Netherlands quite often CT scans were done instead, probably due to limited capacity. In contrast, standard MRI scanning is done in the UK. Another interesting observation was that, additionally to MRI scanning, an examination under anesthesia including rigid sigmoidoscope digital rectal examination is performed pre-operatively in the RMH for optimal surgical planning. It would be interesting to determine the influence of these different ways of pre-operative tumour assessment on oncological outcome and choice of surgical approach in future research.

The main limitation of our study is the relatively short follow-up time compared to the median RFS ( 28 months vs. 75 months). The number of events is therefore low and definite conclusions can only be drawn after analysis of data with longer follow-up. Another problem is the amount of missing data in certain variables, which was addressed by using imputation. Furthermore, it would have been informative to ascertain what proportion of patients initially thought to require an APR, but ultimately had a sphincter sparing procedure after neoadjuvant treatment with imatinib. Nevertheless, this is the largest cohort of European rectal GIST patients to date, and one of the largest in the literature, and it is illustrative to combine all available data on such a rare tumour from countries that all manage patients according to the ESMO guideline.

\section{Conclusions}

This study represents a large cohort of surgically treated rectal GIST patients in Europe. In European clinical practice, smaller tumours are most likely treated by LTR, while the larger tumours are preferentially treated with LAR and APR and patients receive more often perioperative imatinib therapy. LTR, LAR and APR have comparable oncological outcome. Quality of treatment (multimodal approach, less peroperative tumour rupture) appears to be superior in specialist centres and referral of rectal GIST to specialist centres is therefore recommended.

\section{Funding}

A research grant for the Dutch GIST Registry was received from Novartis (3017/13), Pfizer (WI189378) and Bayer (2013-MED12005). These funding sources did not have any involvement in conducting this research.

\section{Declaration of competing interest}

P. Rutkowski has received honoraria for lectures and Advisory Board from Novartis, Pfizer and Blueprint Medicines. The other authors declare that they have no known competing financial interests or personal relationships that could have appeared to influence the work reported in this paper.

\section{Appendix}


Table A1

Patient and tumour characteristics whole cohort of GIST patients.

\begin{tabular}{|c|c|c|}
\hline Characteristic & No. & $\%$ \\
\hline & 210 & 100 \\
\hline \multicolumn{3}{|l|}{ Gender } \\
\hline Male & 132 & 63 \\
\hline Female & 78 & 37 \\
\hline Age (median, range) & $61(18-91)$ & \\
\hline Tumour size at diagnosis in mm (median, range) & $65(3-250)$ & \\
\hline Distance to anal verge in mm (median, range) & $34(0-200)$ & \\
\hline \multicolumn{3}{|l|}{ Most important symptom at diagnosis } \\
\hline Rectal bleeding & 27 & 13 \\
\hline Change in bowel habits & 25 & 12 \\
\hline Rectal mass & 22 & 11 \\
\hline Pain & 16 & 8 \\
\hline Urinary tract problems & 9 & 4 \\
\hline Incidental finding & 9 & 4 \\
\hline Screening program & 2 & 1 \\
\hline Vaginal bleeding & 2 & 1 \\
\hline Not specified & 22 & 11 \\
\hline \multicolumn{3}{|l|}{ Mutation status } \\
\hline KIT exon 11 & 110 & 53 \\
\hline KIT exon 9 & 21 & 10 \\
\hline Wildtype (KIT/PDGFR) & 19 & 9 \\
\hline KIT exon 13 & 5 & 2 \\
\hline PDGFR & 1 & 1 \\
\hline \multicolumn{3}{|l|}{ Mitotic rate at baseline } \\
\hline Low $(\leq 5 / 50 \mathrm{HPF})$ & 63 & 59 \\
\hline High $(>5 / / 50 \mathrm{HPF})$ & 43 & 41 \\
\hline \multicolumn{3}{|l|}{ Surgery yes/no } \\
\hline Yes & 155 & 74 \\
\hline No & 55 & 26 \\
\hline
\end{tabular}

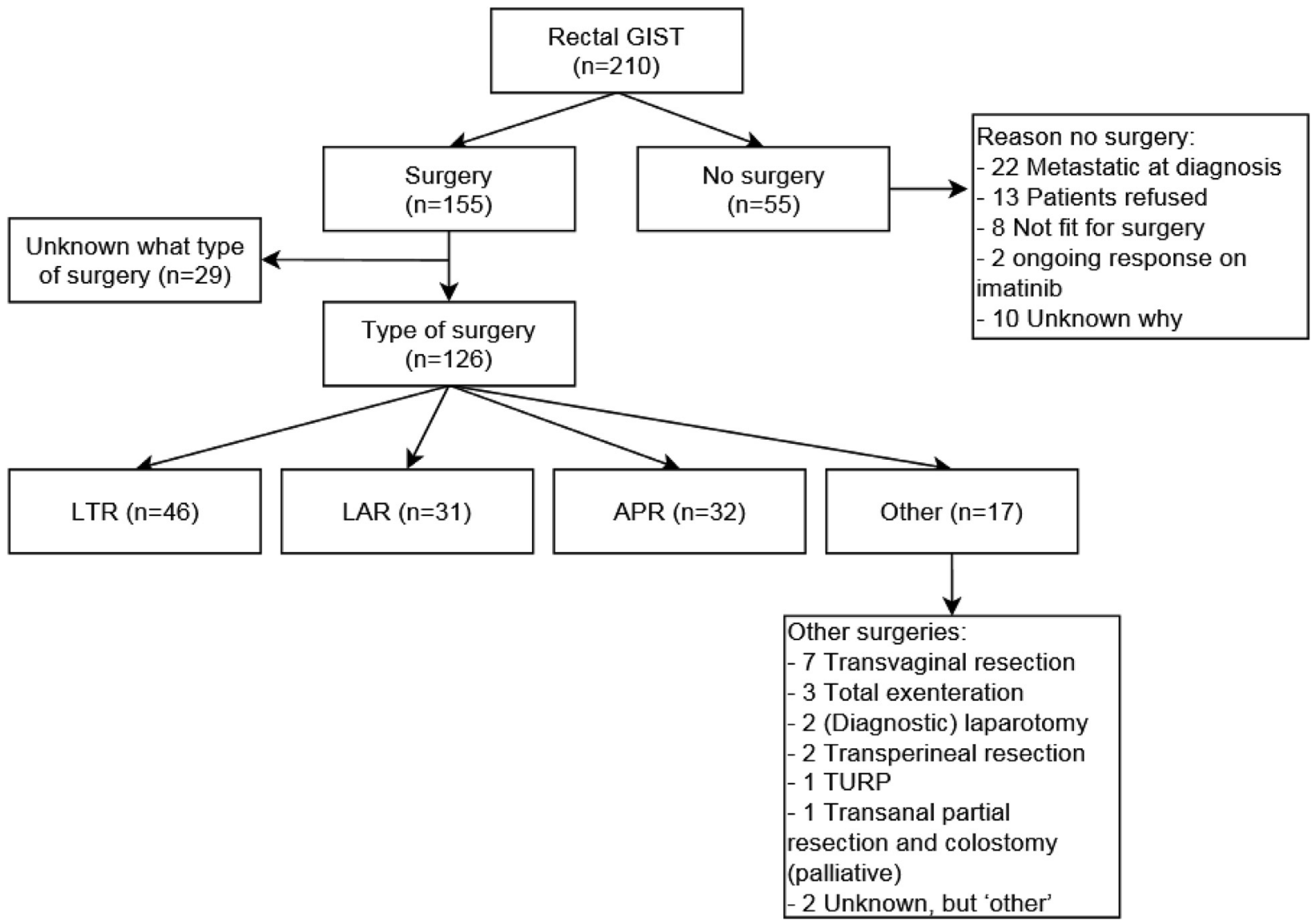

Fig. A1. Flowchart of rectal GIST patients. 
Table A2

Characteristics of rectal GIST patients with three main types of surgery $(\mathrm{n}=109)$.

\begin{tabular}{|c|c|c|c|c|c|}
\hline & No. (\%) & LTR & LAR & APR & p-value \\
\hline Number of patients & 109 & 46 & 31 & 32 & \\
\hline Age (median, range) & $61(27-83)$ & $61(40-82)$ & $62(38-83)$ & $61(27-81)$ & $0.942^{\mathrm{A}}$ \\
\hline Gender & & & & & $0.808^{\mathrm{B}}$ \\
\hline Male & $76(70)$ & 31 & 23 & 22 & \\
\hline Female & $33(30)$ & 15 & 8 & 10 & \\
\hline Baseline size in mm (median, range) & $61(14-250)$ & $50(14-135)$ & $70(20-190)$ & $82(37-250)$ & $<0.001^{\mathrm{A} *}$ \\
\hline Baseline distance to anal verge in mm (median, range) & $35(0-80)$ & $40(10-80)$ & $30(19-60)$ & $30(0-80)$ & $0.083^{\mathrm{A}_{*}}$ \\
\hline Mutation & & & & & $0.533^{\mathrm{B}}$ \\
\hline KIT exon 11 & $68(74)$ & 30 & 19 & 19 & \\
\hline KIT exon 9 & $14(17)$ & 3 & 5 & 6 & \\
\hline KIT exon 13 & $5(5)$ & 2 & 1 & 2 & \\
\hline Wildtype & $5(5)$ & 1 & 3 & 1 & \\
\hline Baseline mitotic rate & & & & & $0.246^{\mathrm{B}}$ \\
\hline Low $(\leq 5 / 50 \mathrm{HPF})$ & $36(57)$ & 16 & 7 & 13 & \\
\hline High $(>5 / / 50 \mathrm{HPF})$ & $27(43)$ & 11 & 10 & 6 & \\
\hline Type of hospital & & & & & $0,001^{\mathrm{B} *}$ \\
\hline Sarcoma centre & $75(69)$ & 23 & 23 & 29 & \\
\hline General hospital & $34(31)$ & 23 & 8 & 3 & \\
\hline Resection margin & & & & & $0.438^{\mathrm{B}}$ \\
\hline R0 & $67(62)$ & 26 & 18 & 23 & \\
\hline $\mathrm{R} 1$ & $31(29)$ & 14 & 11 & 6 & \\
\hline $\mathrm{R} 2$ & $10(9)$ & 6 & 2 & 2 & \\
\hline Peroperative tumour rupture & & & & & $0.351^{\mathrm{B}}$ \\
\hline No & $73(85)$ & 27 & 20 & 26 & \\
\hline Yes & $13(15)$ & 6 & 5 & 2 & \\
\hline Severe complications (>3a Clavien Dindo) & & & & & $0,015^{\mathrm{B} *}$ \\
\hline No & $86(96)$ & 33 & 27 & 26 & \\
\hline Yes & $4(4)$ & 0 & 0 & 4 & \\
\hline Stoma & & & & & $<0.001^{\mathrm{B} *}$ \\
\hline No & $35(32)$ & 30 & 5 & 0 & \\
\hline Yes, protective stoma & $42(39)$ & 16 & 26 & 0 & \\
\hline Yes, definite stoma & $32(29)$ & 0 & 0 & 32 & \\
\hline Time to closure of protective stoma (median, weeks) & $23(5-120)$ & $27(11-73)$ & $15(5-120)$ & NA & $0.085^{\mathrm{C}}$ \\
\hline \multicolumn{6}{|l|}{ Imatinib neoadjuvant } \\
\hline No & $31(28)$ & 21 & 7 & 3 & $0,002^{\mathrm{B} *}$ \\
\hline Yes & $78(72)$ & 25 & 24 & 29 & \\
\hline Imatinib adjuvant & & & & & $0.062^{\mathrm{B}}$ \\
\hline No & $33(32)$ & 17 & 4 & 12 & \\
\hline Yes & $70(68)$ & 27 & 24 & 19 & \\
\hline
\end{tabular}

$* \mathrm{p}<0.05$ is considered significant.

A Kruskal-wallis test

${ }^{\mathrm{B}}$ Chi square test.

${ }^{\mathrm{C}}$ Mann Whitney $U$ test.

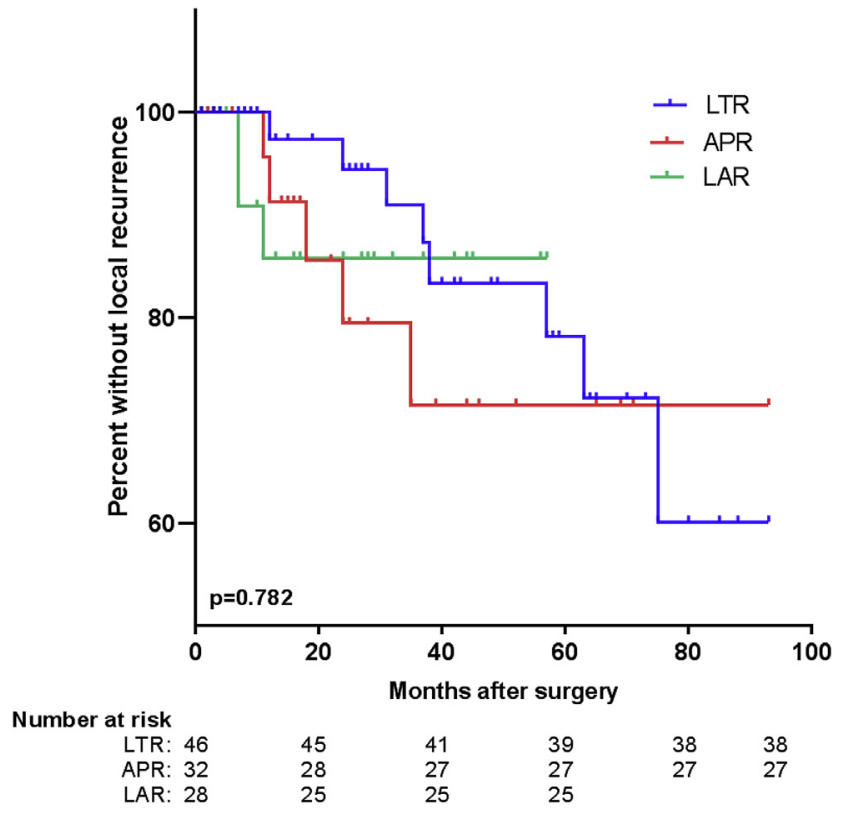

Fig. A2. Local recurrence free survival for three main groups of surgery. 
Table A3

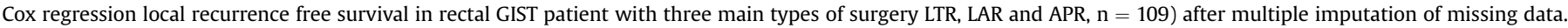

\begin{tabular}{|c|c|c|c|c|c|c|}
\hline & \multicolumn{3}{|c|}{ Univariate analysis } & \multicolumn{3}{|c|}{ Multivariate analysis } \\
\hline & $\mathrm{HR}^{\mathrm{a}}$ & $95 \% \mathrm{Cl}^{\mathrm{b}}$ & p-value & $\mathrm{HR}^{\mathrm{a}}$ & $95 \% \mathrm{Cl}^{\mathrm{b}}$ & P-value \\
\hline \multicolumn{7}{|l|}{ Gender } \\
\hline Male & Reference & & & & & \\
\hline Female & 1.420 & $0.525-3.840$ & 0.490 & & & \\
\hline Age & 1.060 & $1009-1113$ & $0.020 *$ & 1.057 & $1.000-1.116$ & $0.048 * *$ \\
\hline Size & 1.008 & $0.995-1.020$ & $0.234 *$ & 1.006 & $0.992-1.020$ & 0.386 \\
\hline Baseline distance to anal verge & 0.967 & $0.934-1.004$ & $0.084 *$ & 0.976 & $0.934-1.021$ & 0.288 \\
\hline \multicolumn{7}{|l|}{ Mutation } \\
\hline KIT exon 11 & Reference & & & & & \\
\hline No KIT exon 11 & 0.681 & $0.196-2.368$ & 0.546 & & & \\
\hline \multicolumn{7}{|l|}{ Baseline mitotic rate } \\
\hline Low $(\leq 5 / 50 \mathrm{HPF})$ & Reference & & & & & \\
\hline High $(>5 / / 50 \mathrm{HPF})$ & 1.663 & $0.392-7.051$ & 0.487 & & & \\
\hline \multicolumn{7}{|l|}{ Type of surgery } \\
\hline LTR & Reference & & & & & \\
\hline LAR & 1.300 & $0.331-5.114$ & 0.707 & & & \\
\hline APR & 1.478 & $0.478-4.571$ & 0.498 & & & \\
\hline \multicolumn{7}{|l|}{ Resection margin } \\
\hline Ro & Reference & & & & & \\
\hline $\mathrm{R} 1$ & 1.571 & $0.527-4.686$ & 0.418 & 1.310 & $0.363-4.728$ & 0.680 \\
\hline $\mathrm{R} 2$ & 3.492 & $0.880-13.860$ & $0.075 *$ & 2.191 & $0.253-19.016$ & 0.475 \\
\hline \multicolumn{7}{|l|}{ Peroperative tumour rupture } \\
\hline No & Reference & & & & & \\
\hline Yes & 2.136 & $0.546-8.360$ & $0.274 *$ & 1.597 & $0.213-11.977$ & 0.645 \\
\hline \multicolumn{7}{|l|}{ Severe complications } \\
\hline No & Reference & & & & & \\
\hline Yes & 0.864 & $0.003-246.108$ & 0.960 & & & \\
\hline \multicolumn{7}{|l|}{ Stoma } \\
\hline No & Reference & & & & & \\
\hline Yes, protective stoma & 0.767 & $0.223-2.638$ & 0.674 & & & \\
\hline Yes, definite stoma & 1.242 & $0.391-3.946$ & 0.713 & & & \\
\hline \multicolumn{7}{|l|}{ Imatinib neoadjuvant } \\
\hline Yes & Reference & & & & & \\
\hline No & 1.916 & $0.717-5.119$ & $0.195 *$ & 1.562 & $0.496-4.915$ & 0.446 \\
\hline \multicolumn{7}{|l|}{ Imatinib adjuvant } \\
\hline Yes & Reference & & & & & \\
\hline No & 0.887 & $0.291-2.701$ & 0.832 & & & \\
\hline
\end{tabular}

$* \mathrm{p}<0.3$ in univariate and therefore included in multivariate analysis, $* * \mathrm{p}<0.05$ is considered significant in multivariate analysis.

a: Hazard Ratio, b: Confidence Interval.

\section{References}

[1] Søreide K, Sandvik OM, Søreide JA, Giljaca V, Jureckova A, Bulusu VR. Globa epidemiology of gastrointestinal stromal tumours (GIST): a systematic review of population-based cohort studies. Cancer Epidemiol 2016;40:39-46.

[2] Verschoor AJ, Bovée JVMG, Overbeek LIH, Hogendoorn PCW, Gelderblom H, group P. The incidence, mutational status, risk classification and referra pattern of gastro-intestinal stromal tumours in The Netherlands: a nationwide pathology registry (PALGA) study. Virchows Arch 2018;472(2):221-9.

[3] van der Graaf WTA, Tielen R, Bonenkamp JJ, Lemmens V, Verhoeven RHA, de Wilt JHW. Nationwide trends in the incidence and outcome of patients with gastrointestinal stromal tumour in the imatinib era. Br J Surg 2018;105(8): 1020-7.

[4] Miettinen M, Lasota J. Gastrointestinal stromal tumors: pathology and prognosis at different sites. Semin Diagn Pathol 2006;23(2):70-83.

[5] Miettinen M, Lasota J. Gastrointestinal stromal tumors. Gastroenterol Clin N Am 2013:42(2):399-415.

[6] Casali PG, Abecassis N, Aro HT, et al. Gastrointestinal stromal tumours: ESMO EURACAN Clinical Practice Guidelines for diagnosis, treatment and follow-up. Ann Oncol 2018;29(Supplement_4):iv267.

[7] Wilkinson MJ, Fitzgerald JE, Strauss DC et al. Surgical treatment of gastrointestinal stromal tumour of the rectum in the era of imatinib. Br J Surg 2015;102(8):965-71.

[8] Huynh TK, Meeus P, Cassier P, et al. Primary localized rectal/pararectal gastrointestinal stromal tumors: results of surgical and multimodal therapy from the French Sarcoma group. BMC Canc 2014;14:156.

[9] Jakob J, Mussi C, Ronellenfitsch U, et al. Gastrointestinal stromal tumor of the rectum: results of surgical and multimodality therapy in the era of imatinib. Ann Surg Oncol 2013;20(2):586-92.

[10] Tielen R, Verhoef C, van Coevorden F, et al. Surgical management of recta gastrointestinal stromal tumors. J Surg Oncol 2013;107(4):320-3.

[11] Shen C, Chen H, Yin R, et al. Clinicopathologic, surgical characteristics and survival outcomes of rectal gastrointestinal stromal tumors. Neoplasma 2015;62(4):610-7.

[12] Han X, Xu J, Qiu H, Lin G. A novel curative treatment strategy for patients with lower grade rectal gastrointestinal stromal tumor: chemoreduction combined with transanal endoscopic microsurgery. J Laparoendosc Adv Surg Tech 2017;27(6):579-85.

[13] Zhu R, Liu F, Grisotti G, et al. Distinctive features of gastrointestinal stromal tumors arising from the colon and rectum. J Gastrointest Oncol 2018;9(2): 231-40.

[14] Kukar M, Kapil A, Papenfuss W, Groman A, Grobmyer SR, Hochwald SN. Gastrointestinal stromal tumors (GISTs) at uncommon locations: a large population based analysis. J Surg Oncol 2015;111(6):696-701.

[15] Dematteo RP, Gold JS, Saran L, et al. Tumor mitotic rate, size, and location independently predict recurrence after resection of primary gastrointestinal stromal tumor (GIST). Cancer 2008;112(3):608-15.

[16] Hawkins AT, Wells KO, Krishnamurty DM, et al. Preoperative chemotherapy and survival for large anorectal gastrointestinal stromal tumors: a national analysis of 333 cases. Ann Surg Oncol 2017;24(5):1195-201.

[17] Wang SY, Wu CE, Lai CC, et al. Prospective evaluation of neoadjuvant imatinib use in locally advanced gastrointestinal stromal tumors: emphasis on the optimal duration of neoadjuvant imatinib use, safety, and oncological outcome. Cancers 2019;11(3).

[18] Rutkowski P, Gronchi A, Hohenberger P, et al. Neoadjuvant imatinib in locally advanced gastrointestinal stromal tumors (GIST): the EORTC STBSG experience. Ann Surg Oncol 2013;20(9):2937-43.

[19] Tielen R, Verhoef C, van Coevorden F, et al. Surgical treatment of locally advanced, non-metastatic, gastrointestinal stromal tumours after treatment with imatinib. Eur J Surg Oncol 2013;39(2):150-5.

[20] Joensuu H, Eriksson M, Collan J, Balk MH, Leyvraz S, Montemurro M. Radiotherapy for GIST progressing during or after tyrosine kinase inhibitor therapy: a prospective study. Radiother Oncol 2015;116(2):233-8.

[21] Cuaron JJ, Goodman KA, Lee N, Wu AJ. External beam radiation therapy for locally advanced and metastatic gastrointestinal stromal tumors. Radiat Oncol 2013;8:274.

[22] Lolli C, Pantaleo MA, Nannini M, et al. Successful radiotherapy for local contro of progressively increasing metastasis of gastrointestinal stromal tumor. Rare Tumors 2011;3(4):e49.

[23] Gatto L, Nannini M, Saponara M, et al. Radiotherapy in the management of gist: state of the art and new potential scenarios. Clin Sarcoma Res 2017:7:1. 